\title{
Dielectric Constants of Aqueous Solutions of Dextrose and Sucrose
}

\author{
By Cyrus G. Malmberg and Arthur A. Maryott
}

\begin{abstract}
The dielectric constants of aqueous solutions of dextrose and of sucrose prepared from N BS Standard Samples were determined at $20^{\circ}, 25^{\circ}$, and $30^{\circ} \mathrm{C}$ for concentrations up to 50 and 60 percent by weight, respectively. A bridge method was used, and the sources of error involved are considered. The results, relative to a selected value for water, are considered to be accurate to 0.05 percent or better and are expressed in terms of an empirical equation for the purposes of interpolation.
\end{abstract}

\section{Introduction}

Examination of the literature reveals rather poor agreement in the data reported for the dielectric constants of aqueous solutions of dextrose [ $\left[\begin{array}{lll}1 & \text { to } & 3\end{array}\right]^{1}$ and of sucrose (static values) [4 to 18], (high-frequency data) [19 to 27]. Consequently, it seemed desirable to redetermine their static dielectric constants over a range of concentration in the vicinity of room temperature. Inasmuch as highly purified samples of dextrose and sucrose are available as standard samples at the National Bureau of Standards, these solutions may prove useful as secondary standards of dielectric constant having values intermediate between water and some of the more polar organic solvents.

For determinations of dielectric constants of media having an appreciable but not excessively large conductance, a bridge method employing relatively low and variable frequencies, as in this investigation, has certain advantages over resonance methods with regard to flexibility, accuracy, and the analysis and evaluation of errors. Errors such as arise from electrode polarization or lead inductance may be evaluated from measurements at various frequencies or often made negligible by the proper choice of frequency. Connection and calibration errors can be readily minimized by employing a cell of high capacitance. Perhaps the principal disadvantage is the difficulty sometimes experienced in maintaining sufficient stability of cell resistance to permit sensitive capacitance balance at lower frequencies.

\section{Apparatus}

The a-c bridge was of the equal ratio capacitanceconductance type, as indicated in figure 1 . The ratio arms, $\dot{R}_{1}$ and $R_{2}$, were 1,000 -ohm woven resistors (Leeds \& Northrup Type Std. 697-D) matched to within 0.001 percent in resistance. The measuring arm of resistance $R_{4}$ and capacitance $C_{4}$ consisted of a 100,000-ohm Leeds \& Northrup resistance box (Cat. No. 4764-S) in parallel with a calibrated variable condenser, General Radio Co. Type 722-D. To extend the range of capacitance, General Radio Company's type 505 mica condensers were plugged to the terminals of the precision con-

\footnotetext{
1 Figures in brackets indicate the literature references at the end of this pape
}

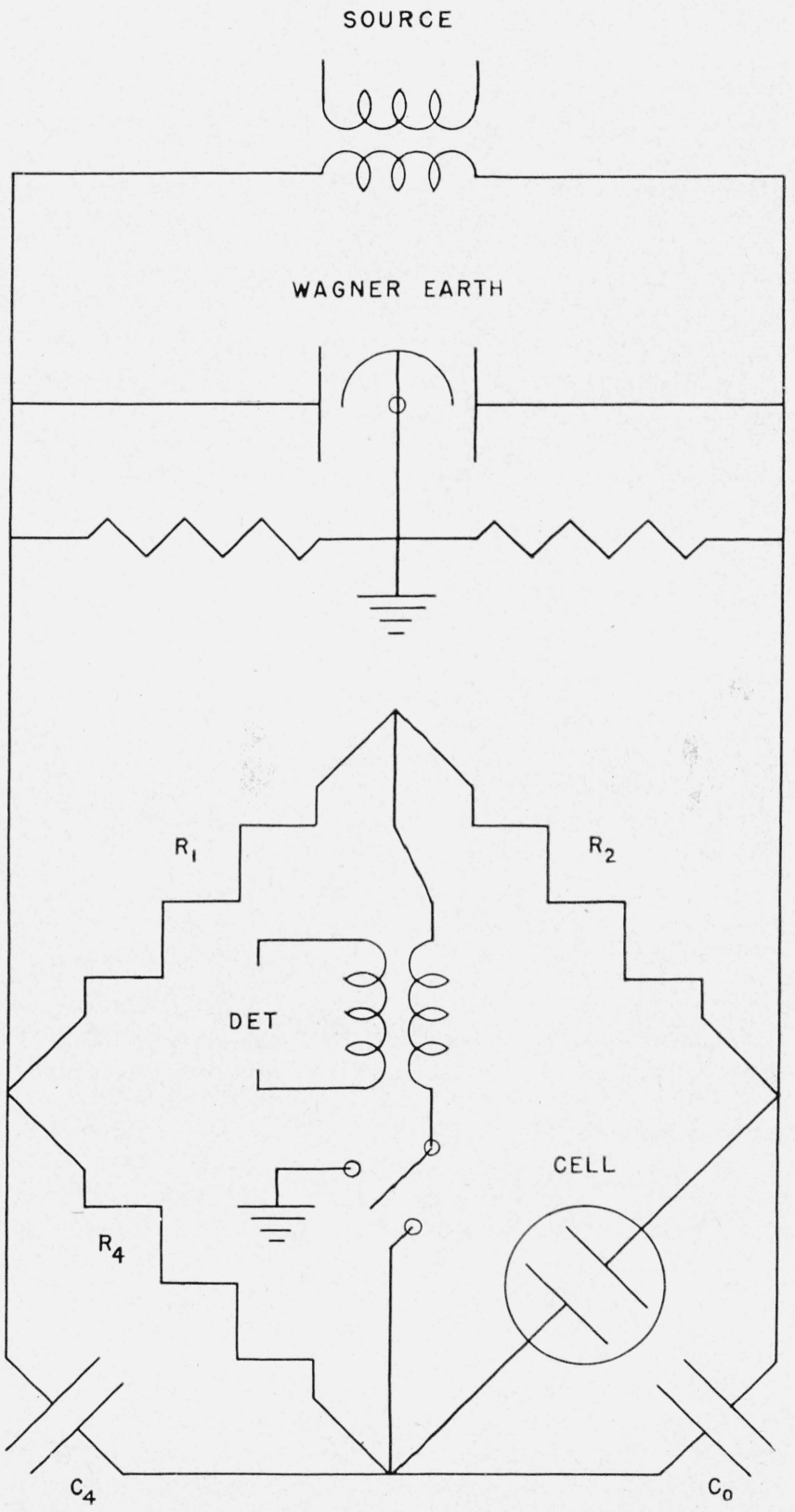

FIgURE 1. Schematic circuit arrangement of capacitanceconductance bridge. 
denser as needed. The mica condensers were calibrated in terms of the precision condenser.

The unknown arm contained the cell and a relatively small variable capacitor, $C_{0}$, in parallel. $C_{0}$ remained fixed throughout the measurements and served only to provide a convenient "zero" setting for $C_{4}$. As no suitable three-terminal cell was available, two unshielded cells of convenient design were employed, each consisting of two concentric cylinders of bright platinum mounted in a glass container. Cell $A$ had a replaceable capacitance of about $30 \mu \mu \mathrm{f}$ and has been described previously [28]. Cell $B$, designed principally for measurements of conductivity, has a replaceable capacitance of approximately $60 \mu \mu \mathrm{f}$ and differed from cell $A$ in that it had parallel rather than concentric leads. In

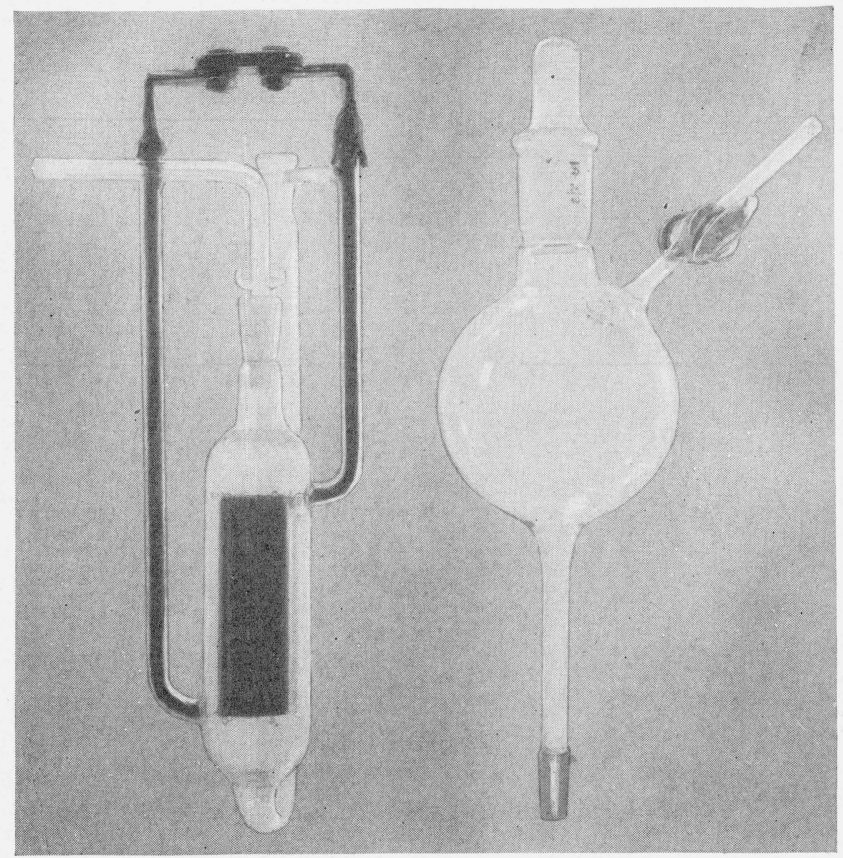

Figure 2. Cell B with dismounted reservoir.

Length of electrodes is $77 \mathrm{~mm}$, separation $1 \mathrm{~mm}$, and the diameter of the outer electrode $28 \mathrm{~mm}$.

addition to the electrode compartment, each cell contained a reservoir to accommodate excess liquid and to permit mixing by forcing the contents back and forth with compressed gas. The construction of cell $B$ is shown in figure 2 with its reservoir dismounted. Concentric mounting of its electrodes was accomplished by sealing to glass beads corresponding pairs of the six small platinum wires, which were welded to each end of the cylinders. The beads were then sealed to the outer walls of the cell. Two of the wires were sealed through the beads to provide contact to the mercury leads.

The cells were mounted in a reproducible position in the grounded thermostat and connected to the bridge terminals, using parallel leads of constant capacitance and inductance. Capacitance balance of the bridge was consistent to $0.1 \mu \mu \mathrm{f}$ on replacement of the cell in the bath and reconnection to the bridge.
The outer electrode was maintained at earth potential through the use of a Wagner earth. The use of this device along with appropriate shielding of the other branches of the bridge was effective in eliminating or fixing the influence of earth admittance and interbranch couplings associated with these branches and the corners of the bridge. Consequently, with the exception of a residual effect due to distributed interelectrode admittance to earth, the measured impedances of the cells were independent of the influence of earth admittances. On the basis of the construction of these cells and their measured earth capacities, this effect was estimated to be no greater than $0.2 \mu \mu \mathrm{f}$ and therefore negligible.

A tuned circuit oscillator variable from 30 cycles to $200 \mathrm{kc}$ was used as the source. A three-stage resistance-coupled amplifier and headphones were used as a detector up to $12 \mathrm{kc}$. At higher frequencies the amplifier was replaced by a Sargent model 11-XA regenerative receiver tuned to give any convenient audible beat-frequency. Throughout the measurements the sensitivity of capacitance balance was 0.1 $\mu \mu \mathrm{f}$ or better. Both source and detector were isolated from the bridge proper by shielded transformers.

Measurements were made at $20^{\circ}, 25^{\circ}$, and $30^{\circ} \mathrm{C}$ with the cell in an oil thermostat controlled to within $0.01^{\circ}$.

\section{Materials and Solutions}

Solutions of sucrose of the desired concentrations were prepared on a weight basis from NBS Standard Sample 17 and conductivity water. Solutions of dextrose were prepared similarly from Standard Sample 41 and were then allowed to stand at least 4 hours in order to establish equilibrium with respect to mutarotation. Freshly prepared solutions of dextrose $(\alpha$-form) had decidedly higher dielectric constants than the equilibrium mixtures. Conductivity water was obtained from ammonia-free distilled water by redistillation. It had a conductivity of about $10^{-7}$ mho before exposure to the atmosphere.

Initial attempts to determine the dielectric constants of the solutions of dextrose were unsuccessful because of the very rapid and continued increase in conductance that occurred when the solutions were introduced into the cell. Overnight the conductance sometimes increased by as much as a hundredfold. As this change was accompanied by a pronounced decrease in $\mathrm{pH}$ and occurred only when the solution was in contact with the electrodes, catalytic oxidation was suspected. Replacement of air by hydrogen eliminated this instability. Consequently, hydrogen was used to flush out the cell, to saturate each solution, and to transfer the solution from the flask to the cell. The same procedure was adopted for the solutions of sucrose because of the advantage of lower conductances gained by removal of dissolved $\mathrm{CO}_{2}$. The specific conductivities of the solutions were $3 \times 10^{-6}$ mho or lower in all cases.

\section{Procedure}

The dielectric constants were determined relative to water at $25^{\circ}$, using the value reported by Wyman 
[29], namely, 78.54. The replaceable vacuum capacitance of the cell, $C_{V}$, was obtained from the difference in capacitance of the cell first in air and then when filled with water, $C_{W}-C_{A}$, respectively, according to

$$
C_{V}=\left(C_{W}-C_{A}\right) / 77.54
$$

The dielectric constant of the unknown medium, $\epsilon_{X}$, is then

$$
\epsilon_{X}=78.54-\Delta C_{X} / C_{V},
$$

where $\Delta C_{X}$ is the change in capacitance of the cell on replacing the unknown medium by water at $25^{\circ}$.

Before the experimental procedure is discussed in any detail, the more significant sources of error will be considered briefly. These errors are associated for the most part with residual impedances in the arms of the bridge network. To a sufficient degree of approximation, the bridge may be simulated by the network illustrated in figure 3 . Reactance in the ratio arms is represented by the equivalent parallel capacitances, $C_{1}$ and $C_{2}$. $\quad L_{M}$ and $L_{X}$ are, respectively, the self-inductances of the leads to the precision condenser and to the cell. The effective series inductance of the resistance box is represented by $L_{4}$ and the resistance of the cell by $R_{X}$. During the course of the measurements, $C_{M}$ and $R_{4}$, and consequently $L_{4}$, are varied. The observed change in capacitance of the precision condenser, $\Delta C_{M}$, is not generally equal to $\Delta C_{X}$ but related according to

$$
\Delta C_{M}=\Delta C_{x}+\Delta C_{a}+\Delta C_{b}+\Delta C_{c}+\Delta C_{d}
$$

where, on neglecting terms of higher order,

$$
\begin{aligned}
& \Delta C_{a}=\left(C_{1}-C_{2}\right)\left(R_{1} / R_{4}-R_{1} / R_{4}^{\prime}\right), \\
& \Delta C_{b}=\frac{L_{4}}{R_{4}^{2}}-\frac{L_{x}}{R_{4}^{2}}-\frac{L_{4}^{\prime}}{\left(R_{4}^{\prime}\right)^{2}}+\frac{L_{x}}{\left(R_{4}^{\prime}\right)^{2}}, \\
& \Delta C_{c}=\omega^{2}\left(L_{x}-L_{M}\right)\left(C_{M}+C_{M}^{\prime}\right) \Delta C_{M},
\end{aligned}
$$

$\omega$ being the angular frequency. The primed symbols refer to solution in the cell and the unprimed to water. $\Delta C_{a}$ represents the effect of reactive asymmetry of the ratio arms; $\Delta C_{b}$, the effect of inductance in series with resistance in the measuring and unknown arms; $\Delta C_{c}$, inductance in series with capacitance in the measuring and unknown arms, and $\Delta C_{d}$ the change in capacitance attributable to electrode polarization.

The effect of polarization capacitance, $C_{d}$, was found to be proportional to $\omega^{-n}$, where $n<2$ and constant for a given cell and solution. On the other hand, $C_{c}$ is proportional to $\omega^{2}$, so the influence of these frequency dependent errors was readily determined by varying the frequency between 3 and $96 \mathrm{kc}$. Actually, in this investigation it was not necessary to extrapolate out these errors, as they became quite negligible at some intermediate frequency where $C_{M}$ reached its minimum value. This frequency was usually $48 \mathrm{kc}$ for the solutions of dextrose (cell $A$ ) and $24 \mathrm{kc}$ for the solutions of sucrose (cell $B$ ).

The frequency-independent errors, expressed as the

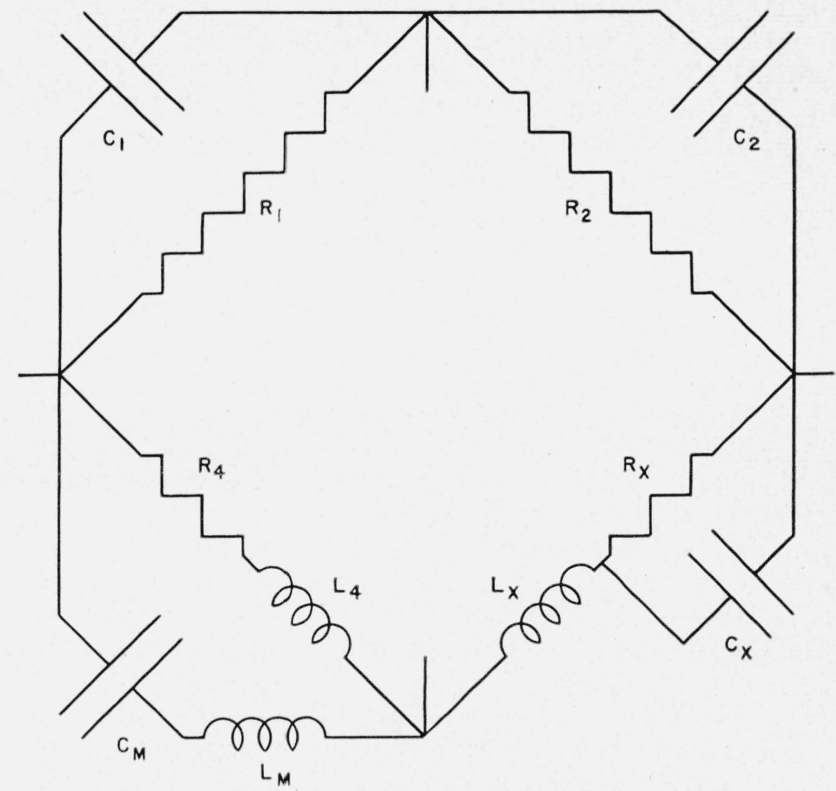

FIGURE 3. Simulated circuit of the bridge showing distribution of effective impedances in the arms of the bridge.

sum $\Delta C_{a}+\Delta C_{b}$, were eliminated by a procedure that was essentially equivalent to making all measurements at the same resistance. With the cell filled with water at constant temperature, its resistance was varied to correspond to the resistances of the solutions by increasing or decreasing the $\mathrm{CO}_{2}$ content. In this manner the change in measured capacitance accompanying a given change in cell resistance was determined. The resistances of the solutions were usually between 1,000 and 3,000 ohms, so that correspondence of the 1,000- and 100-ohm dials only was needed. These corrections were relatively small and amounted to 0.1 unit or less in dielectric constant. The maximum concentration of carbon dioxide introduced into the water during the course of this determination is estimated to be about $2 \times 10^{-4}$ mole/liter. Considering both the ionized and unionized portions, estimates of the effect of this amount of carbon dioxide on the dielectric constant indicate a negligible change, of the order of 0.003 unit or less.

Values of $C_{V}$ for both cells were determined with a known fixed resistor, $R_{A}$, directly across the arm containing the cell both when empty and when filled with water at $25^{\circ} \mathrm{C}$. Because of this shunt (since $R_{x}$ is no longer equal to $R_{4}$ ), the relation of $\Delta C_{x}$ to $\Delta C_{M}$ given in eq 3 is altered with regard to the term $\Delta C_{b}$ with the result that now

$$
\Delta C_{b}=\frac{L_{4}}{R_{4}^{2}}-\frac{L_{x}}{R_{x}^{2}}-\frac{L_{4}^{\prime}}{R_{A}^{2}},
$$

where $R_{x}=R_{4} R_{A} /\left(R_{A}-R_{4}\right)$. Since for corresponding values of $R_{4}$, the difference between this value of $\Delta C_{b}$ and that for direct measurement involves only terms in $L_{x}$ and known values of $R, L_{X}$ was evaluated, and the experimentally determined values of $\Delta C_{a}+\Delta C_{b}$ were corrected as needed for use in calculating $\Delta C_{X}$ to obtain $C_{V}$. 
Although neither cell contained insulating supports between the electrodes, their construction did not preclude some nonlinearity in the variation of capacitance with dielectric constant of the medium. That no detectable error was encountered in this range of dielectric constant was indicated by intercomparisons between the two cells and by a determination of the variation of $C_{V}$ for cell $B$ by using liquids, the dielectric constants of which were determined by an absolute method.

\section{Results}

The experimental data, including those for water at $20^{\circ}$ and $30^{\circ} \mathrm{C}$, are listed in table 1 . Inasmuch as the actual concentrations were very close to even values of weight percent, the dielectric constants have been interpolated to even values. All of the data for the dextrose solutions can be expressed by the equation,

$\epsilon($ dextrose $)=\epsilon_{w}-\left[0.227-1.2\left(10^{-3}\right)(t-25)\right] C$

$$
\begin{aligned}
& -\left[1.32\left(10^{-3}\right)+1.2\left(10^{-5}\right)(t-25)\right] C^{2} \\
& -\left[3.9\left(10^{-6}\right)-2.8\left(10^{-7}\right)(t-25)\right] C^{3}
\end{aligned}
$$

where $\epsilon$ (dextrose) and $\boldsymbol{\epsilon}_{w}$ are, respectively, the dielectric constants of the dextrose solution and of water at the temperature, $t$, in degrees Celsius, and $C$ is the weight percent of dextrose. This equation fits the data of table 1 with an average deviation of 0.02 and a maximum deviation of less than 0.04 unit in dielectric constant. For the solutions of sucrose, the corresponding equation,

$$
\begin{gathered}
\epsilon(\text { sucrose })=\epsilon_{w}-0.226 C-\left[6.75\left(10^{-4}\right)-1.5\left(10^{-5}\right)\right. \\
(t-25)] C^{2} \\
-\left[1.09\left(10^{-5}\right)+4\left(10^{-8}\right)(t-25)\right] C^{3},
\end{gathered}
$$

fits the data with an average deviation of 0.01 and a maximum deviation of less than 0.03 unit. Exclu-

TABLE 1. Dielectric constants of aqueous dextrose and sucrose solutions

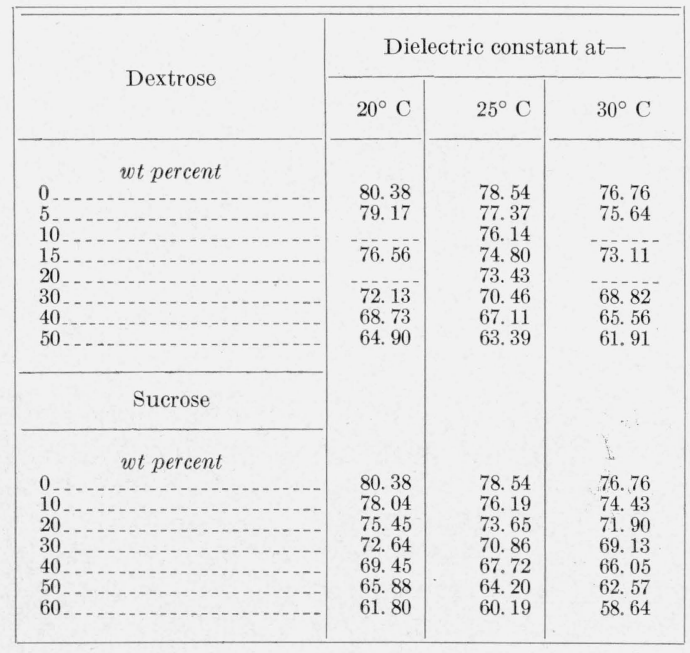

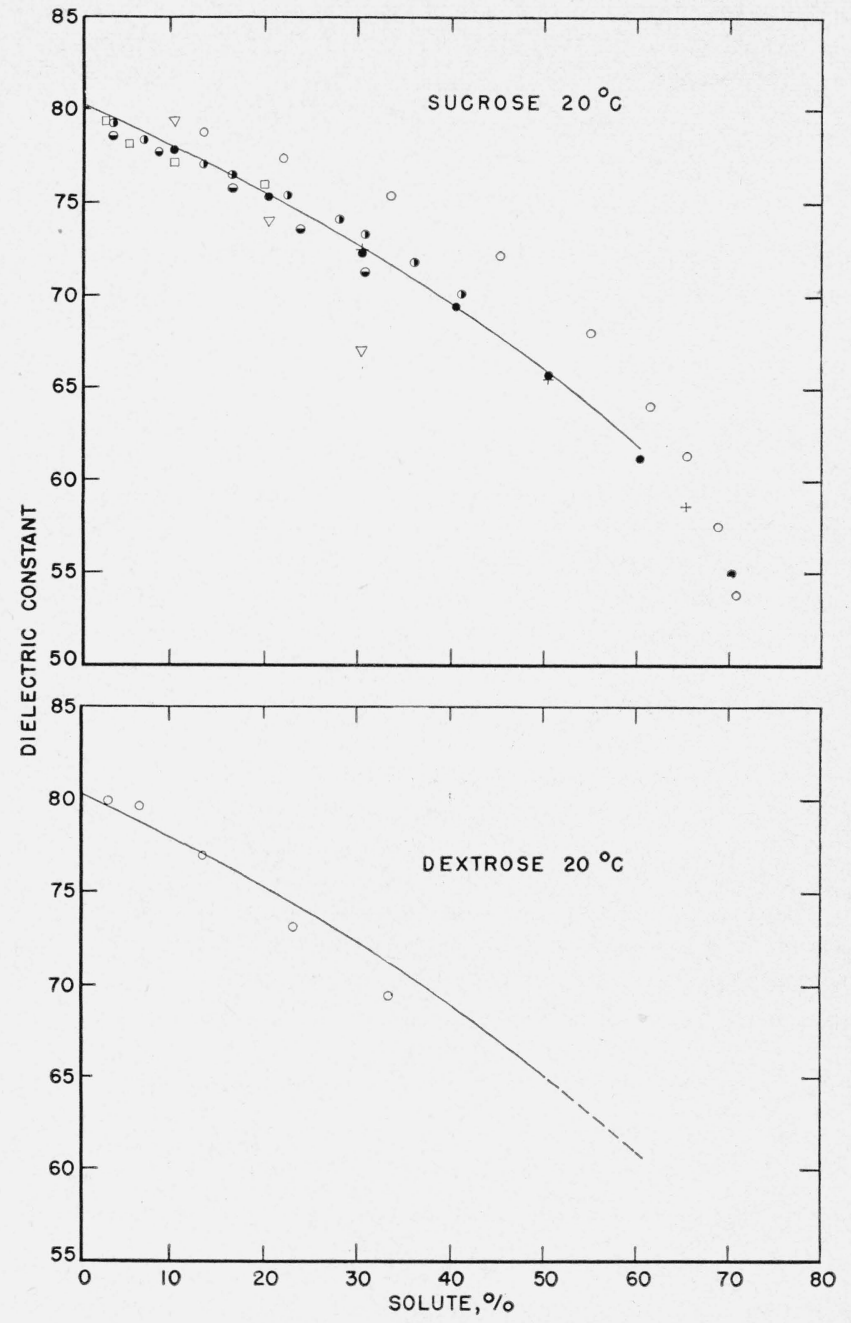

Figure 4. Comparison of dielectric constant data reported on aqueous solutions of dextrose and sucrose.

: Kockel-10, 20, 30, and 40 percent; $600 \mathrm{kc}$. Davies - 10 , and 20 percent; 1.5 to 4 mc. Kniekamp-10 and 20 percent; 566 kc. Akerlöf-50, 60, and 70 percent; $2 \mathrm{mc}$. These data are plotted under a common symbol, as the differences in value of $\epsilon$ reported could not be resolved for the purposes of this graph. 0 , Berline and Rüter $-3.3 \mathrm{mc}$; Harrington $-1 \mathrm{mc} ; \square$, Kistler $-2.1 \mathrm{mc} ; \nabla$, Furth $-400 \mathrm{mc}$ , Astin $-2.7 \mathrm{mc} ;+$, Slevogt $-3.1 \mathrm{mc} ;$
_

sive of any uncertainty in the value selected for water, these data are probably accurate to 0.05 percent or better.

A comparison of these results at $20^{\circ} \mathrm{C}$ with those of some other workers after correction to the same dielectric constant for water is given in figure 4 . The curves represent the present data, and that for dextrose is extrapolated to 60 percent for comparative purposes. Of the data on dextrose, only those of Berliner and Rüter obtained at $3.3 \mathrm{mc}$ are comparable to the present data as representing static values, and the agreement is poor. For the data on sucrose the best agreement is shown by those of Kockel, Akkerlöf, Kniekamp, Slevogt (at $3.1 \mathrm{mc}$ ), and to a lesser extent by those of Davies as reported by Lattey, Gatty, and Davies. These values are, in general, lower than the present data by from 0.1 to 1 percent and were obtained by resonance methods. 
Berliner and Rüter also used a resonance method. Astin developed and used a method employing voltage resonance that was subsequently adopted by Akerlöf. The values reported by Harrington were obtained by means of a bridge method at $1 \mathrm{mc}$, and it is uncertain what method Kistler used at $2.1 \mathrm{mc}$. The measurements of Furth $(400 \mathrm{mc})$ and others [19 to 27] were made at very high frequencies, so the low values reported are undoubtedly attributable to anomalous dispersion and are not comparable to static values.

Consideration of the data available on static values suggests that the major descrepancies in the values reported are in large part due to high conductivity as, in general, it appears that precautions have not been taken to obtain and maintain low conductivities for these solutions. To a lesser extent it is also probable that frequency-dependent errors are associated with many of these values. The extent to which these factors may modify the suitability of a method of measurement is exemplified by Kniekamp's [9] study and Hartshorn's analysis [30] of resonance methods.

\section{References}

[1] E. Berliner and R. Rüter, Kolloid-Z. 47, 251 (1929).

[2] R. Furth, Ann. Physik. 70, 63 (1923).

[3] G. Kreinina, J. Exptl. Theoret. Phys. (USSR) 15, 208 (1945).

[4] G. Akerlöf, J. Am. Chem. Soc. 54, 4125 (1932).
[5] A. V. Astin, Phys. Rev. 34, 300 (1929).

[6] E. Berliner and R. Rüter, Kolloid-Z. 47, 251 (1929).

[7] E. H. Harrington, Phys. Rev. 8, 581 (1916).

[8] S. S. Kistler, J. Phys. Chem. 35, 815 (1931).

[9] H. Kniekamp, Z. Physik 51, 95 (1928).

[10] L. Kockel, Ann. Physik 78, 417 (1925).

[11] E. Landt, Centr. Zuckerind. 44, 723 (1936).

[12] R. T. Lattey, Phil. Mag. 41, 829 (1921).

[13] R. T. Lattey, O. Gatty, and W. G. Davies, Phil. Mag. 12, 1019 (1931).

[14] H. Possner, Ann. Physik 6, 815 (1930).

[15] G. Scatchard, J. Am. Chem. Soc. 48, 2026 (1926).

[16] C. Schreck, Physik. Z. 37, 156 (1936).

[17] K. E. Slevogt, Ann. Physik. 36, 141 (1939).

[18] P. Walden and O. Werner, Z. physik. Chem. 129, 405 (1928).

[19] M. Von Ardenene, O. Groos, and G. Otterbein, Physik. Z. 37, 533 (1936).

[20] W. Dahms, Ann. Physik 26, 177 (1936).

[21] O. Dobenecker, Ann. Physik 17, 699 (1933).

[22] P. Drude, Z. physik. Chem. 23, 267 (1897).

[23] A. Duebner, Ann. Physik 84, 429 (1927).

[24] R. Furth, Ann. Physik 70, 63 (1923).

[25] R. Keller, Kolloid-Z. 29, 193 (1921).

[26] G. Kreinina, J. Exptl. Theoret. Phys. (USSR) 15, 208 (1945).

[27] P. Wenk, Ann. Physik 17, 679 (1933).

[28] A. A. Maryott, J. Research NBS 38, 527 (1947) RP1794.

[29] J. Wyman, Phys. Rev. 35, 623 (1930).

[30] L. Hartshorn, Radio frequency measurements by bridge and resonance methods (John Wiley \& Sons, Inc., New York, N. Y., 1940).

Washington, April 13, 1950

\title{
Response of Accelerometers to Transient Accelerations
}

\author{
By Samuel Levy and Wilhelmina D. Kroll
}

\begin{abstract}
Curves and tables are shown for the response of accelerometers to transient exciting accelerations. Three types of acceleration-time relations are considered. When plotted, they have square, triangular, and half-sine-wave shapes. The natural periods of the accelerometers for which the computations were made were approximately one, one-third, and one-fifth of the duration of the acceleration pulse. The damping coefficients of the accelerometers were $0,0.4,0.7$, and 1.0 times the critical values. It is indicated that, to obtain an accuracy of better than 5 percent of the peak acceleration in measuring acceleration pulses having the general characteristics of the triangular or sinusoidal pulses, an accelerometer must have a natural period of about one-third the duration of the acceleration pulse, and a damping constant of about 0.4 to 0.7 of the critical value.
\end{abstract}

\section{Introduction}

Accelerometers are widely used to measure oscillatory and transient vibrations.

The fidelity with which these instruments respond in the case of oscillatory stimuli has been thoroughly studied [1, pp. 61 to 70$]$. It is found that, when the damping is between 0.6 and 0.7 of the critical value and the natural period of the accelerometer is less than about half of the period of the applied acceleration, the accuracy is satisfactory.

In the case of excitation of the accelerometer by a transient vibration, only scattered information is available regarding the reliability of the response obtained. Weiss [2] gives the response to a tri- angular pulse of acceleration for an accelerometer whose natural period is 0.3 the duration of the pulse and whose damping is $0,0.3$, and 0.7 of the critical value. He also gives the response to a suddenly applied constant acceleration for accelerometers with a damping ratio of $0,0.3,0.7$, and 1.0 times the critical value. Welch [3] has determined, on the Westinghouse transient analyzer, the response to several kinds of impulses of a $50 \mathrm{c} / \mathrm{s}$ single-degree-offreedom shock measuring instrument having various amounts of damping. On the basis of these scattered data, and information for undamped accelerometers derived by Frankland [4], Biot and Bisplinghoff [5], and others, it has been common practice to assume that an accelerometer will be acceptable in a given 